\title{
Establishing Optimal Mental Health Care for Common Mental Disorders in Primary Health Care
}

\author{
Lauren Kennedy ${ }^{\mathrm{a}}$, Rachelle Ashcroft ${ }^{\mathrm{b}}$
}

\begin{abstract}
Various definitions of health and mental health exist, however there is a generally persistent inclusion and acknowledgement of the importance of holistic elements such as environment and relationships. Integration of the physical, social, and mental aspects of an individual, through the social determinants of health is an important component in establishing the effective delivery of optimal mental health care. With increasing numbers of collaborative care teams, and mental health promotion strategies, primary health care is increasingly building its capacity to help respond to these holistic mental health care needs, with increased and more purposeful attention to the social determinants of health. Despite these steps in the right direction, a gap continues to exist in the delivery of mental health care and many people continue to struggle in accessing adequate treatment. In order to determine how best to proceed, it is important to understand what mental health is, what mental health care in primary health care looks like, what the existing challenges to the delivery of mental health care in primary health care are, and what other models have been successful in integrating the social determinants of health and mental health into the primary health care system.
\end{abstract}

Keywords: mental health, primary health care, common mental disorders, depression, anxiety, social determinants of health

Depression and anxiety - also referred to as common mental disorders (CMDs) - are the most frequent mental health concern encountered in the primary health care setting and represent a serious public health concern (Primary Care Companion, 2008). There is a need for further developing effective treatment and management strategies in order to address the growing burden of disease presented by common mental disorders (Whiteford et al., 2013). There is international support to improve the capacity of primary health care to address prevention and treatment needs associated with common mental disorders (Whiteford et al., 2013). As a social work student, this topic is of importance not only as a means to providing optimal care for individuals that we work with, but also because the particular focus and attention the field of social work places on factors such as the social determinants of health lends itself greatly to this conversation.

Primary health care refers to the first level of care and typically the initial point of contact individuals have with the health care system (Health Council of Canada, 2005). The World Health Organization (WHO) goes further to describe it as being both a philosophy and a model for providing health care services. Effective primary health care is communitybased, is engaged in health promotion, provides continuing care, and recognizes the importance of the social determinants of health (Canadian Institute for Health Information (CIHI), 2006).

There is a general growing level of interest amongst family physicians toward providing better mental health care for example, with common mental disorders (CMHA, 2012; Clatney, MacDonald, \& Shah, 2008). This could be as a result of the growing awareness of the effects mental disorders such as depression and anxiety have not only on individuals and their families, but also on society as a whole. We are also witnessing rising rates of depression, paired with a frequently inadequate current model of treatment within primary health care (Craven \& Bland, 2013). Improving capacity of primary health care is an important first step in improving our current models of care, and moving toward a more effective approach to the treatment of common mental disorders. However, despite this increased focus and initiatives to enhance mental health care (CMHA, 2015; CIHI, 2009; CAMH, CHP, CMHA, Health Nexus, OPHA, 2008), there is still a general dissatisfaction amongst family physicians with the quality of mental health care they are able to provide (Clatney, MacDonald, \& Shah, 2008; Whitebird et al., 2013). For example, treatment of common mental disorders within primary health care settings has proven to be significantly more time consuming relative to the treatment of other health concerns (Culpepper et al., 2006), which is more costly to manage and has been challenging for family physicians who have an excess of daily clinical demands (Kendrick, 2013).

Newer collaborative models of primary health care such as Family Health Teams in Ontario, Primary Care Networks in Alberta, and Family Medicine Groups in Quebec intend to increase capacity for providing treatment and case management for those patients experiencing common mental disorders. These interprofessional health care teams provide patients with increased access to services and expertise from a diverse range of fields such as counselling, pharmacy, nutrition, etc. They are also generally more community-based and flexible in their care delivery models (Mitchell et al., 2012). Successful implementation of these collaborative models of care in Canada and other countries have demonstrated improved clinical outcomes, more effective and efficient use of resources, and improved patient experiences in seeking and receiving care (Kates et al., 2011). However there is still a ways to go before optimal mental health care has been reached. Despite efforts to improve mental health care in primary health care settings, a gap continues to exist and many people continue to struggle in accessing adequate treatment (Kates et al., 2011). In order to understand treatment needs for common mental disorders, this paper seeks to first explore what is mental health in the broadest terms, and how we conceive it; following which, explores mental health care within primary health care settings, highlighting some of the existing challenges in the delivery of quality mental health care within primary care. This paper aims to demonstrate the broad ways in which mental health can be understood, and seeks to

a. Renison School of Social Work, University of Waterloo, Canada

b. Assistant Professor, Factor-Inwentash Faculty of Social Work, University of Toronto, Canada 
encourage consideration of ways that primary health care can persist beyond the existing challenges in mental health care delivery in order to be inclusive of a more holistic approach to care.

\section{What is Mental Health?}

There is presently no universally accepted definition of what mental health is, and various influential bodies each have their own standard definitions. When examining these various definitions, it is interesting to note a persistent and evident inclusion of holistic elements such as environment and relationships. The World Health Organization (WHO) defines mental health as being a state of well-being whereby an individual is able to cope with normal life stresses, to work productively and gainfully, and is able to contribute to their community (WHO, 2014). The Public Health Agency of Canada adds to this definition the inclusion of a sense of emotional and spiritual well-being that acknowledges the importance of culture, equity, social justice, interconnections, and personal dignity (Government of Canada, 2006). The Canadian Mental Health Association (CMHA) declares mental health to entail striking a balance between all aspects of an individual's life, including social, physical, spiritual, economic, and mental realms. They describe this balancing act as a learning process whereby each individual has their own unique challenges to maintaining that balance and staying mentally healthy (CMHA, 2015). Although some differences exist between these definitions, there appears to be a general agreement that mental health is a holistic concept which requires the balance and integration of physical, social, and mental aspects of an individual.

In order to fully understand this balance, we also need to take a step back and understand the distinction between the definitions of "health" versus "mental health". The most commonly quoted definition of health comes from the World Health Organization, and states that health is "a complete state of physical, mental and social well-being, and not merely the absence of disease or infirmity" (WHO, 2006). Overall health is commonly perceived to go beyond the absence of disease, to also include a state of balance within and between oneself and one's physical and social environment that allows full functioning (Sartorius, 2002). Both of these definitions however pose a problem in the absoluteness of the terms of wellbeing, and lend to the further medicalization of society, and to limitations in our understanding of health (Godlee, 2011; Huber et al, 2011; Manwell et al., 2015). Maslow's (1968) influential model demonstrates the historical recognition that food, shelter, survival, protection, society, social support, and freedom from pain, environmental hazards, unnecessary stress, and from any exploitation are integral to health. The recognition of broad influences, like shelter and environment, on health has been clearly made (Marmot et al., 2012; Braveman, Egerter, \& Mockenhaupt, 2011). We see through these definitions that there is an interwoven relationship between physical health and mental health. Health in general is affected by biological, social, psychological, and environmental factors. While mental health is also affected by these factors, it can be a more fluid concept which has an even harder time fitting into the presently accepted absolute measures of health.

An individual's mental health experience may be influenced by internal or external factors. For example, an individual may be more susceptible to mental health demotion or as a result of internal factors such as lack of emotional resilience, low self-esteem, low social status, feelings of helplessness, and problems associated with sexuality, isolation, or lack of integration (Bhugra, Till, \& Sartorius, 2013). Mental health challenges can also be precipitated by external factors such as but not limited to, poor social conditions, discrimination or abuse, conflicts of culture, stigma, or lack of self-determination (Bhugra, Till, \& Sartorius, 2013). A positive state of mental health is when the individual maintains the ability to form and maintain relationships, to perform in culturally appropriate social roles, to identify and communicate positive thoughts and behaviours, and to manage and regulate emotions (Society for Health Education and Promotion Specialists (SHEPS), 1997). These factors further the notion that good mental health is a state of equilibrium in which the individual is at peace with themselves, and is able to fulfill their needs while functioning effectively socially. Focusing on the social determinants of health lends greatly to this balancing process, and has been found to be particularly helpful when providing care for common mental disorders (Raphael, 2009).

Following this idea of maintaining a balance, there are several ways of measuring and gauging mental health. One such way is along one straight continuum where optimal mental health is represented on one end and poor mental health including mental disorders represents the other, with mental health problems falling somewhere in between these respective ends (Alberta Health and Wellness, 2006; Lakaski, 2006) (Figure 1). Another means of measurement is two intersecting continua where we have the same first continuum mentioned, but also a second going from no symptoms of mental illness, to serious mental illness. This provides us with a grid of four quadrants representing optimal mental health and mental illness, optimal mental health and no symptoms of mental illness, poor mental health and mental illness, and poor mental health and no symptoms of mental illness (Minister of National Health and Welfare, 1988; Lakaski, 2006; Canadian Mental Health Association, 2006) (Figure 2). The third approach takes the most holistic standpoint to gauging mental health in that it utilizes a circular model for promoting well-being and overall quality of life. One part of the circle represents the prevention, treatment, and maintenance of mental illness, while the other part represents aspects of mental health promotion such as competence, resilience, empowerment, and supportive environments (Barry, 2001; CIHI, 2009) (Figure 3). This holistic framework fits well with the increasingly prominent concept of the social determinants of health. 


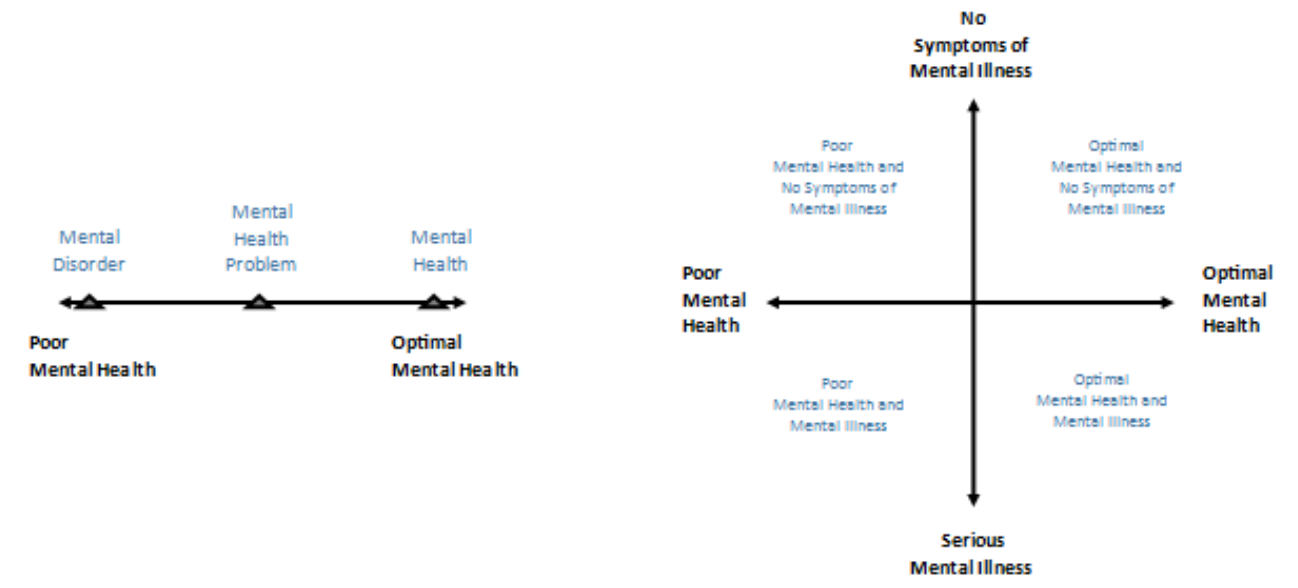

Figure 1.
Mental Health Continuum

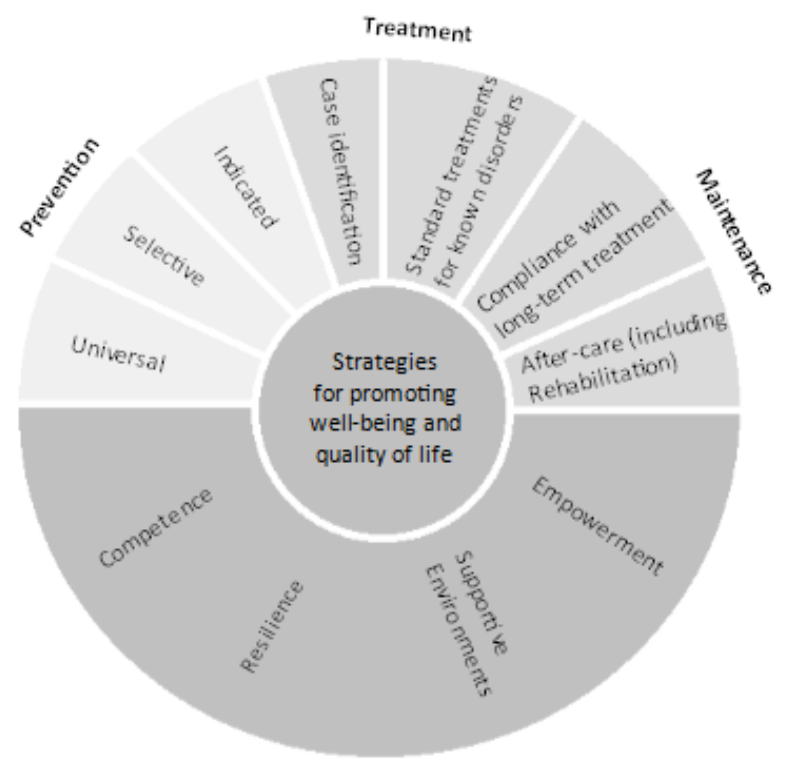

Mental health promotion

These determinants include various social factors that influence health and mental health positively and negatively. They include factors such as income and income distribution, education, unemployment and job security, employment and working conditions, early childhood development, food insecurity, housing, social exclusion, social safety network, access to health services, aboriginal status, gender, race, and disability (Mikkonen \& Raphael, 2010).
The last third model of measurement (Figure 3) presented best represents the direction increasingly being taken in addressing mental health through acknowledging and adhering to factors such as the social determinants of health and taking a more holistic approach to health care.

\section{Primary Mental Health Care}

Primary health care is the leading point of contact for individuals seeking mental health care. Over half of Canadians 
utilizing health care services for mental health concerns received this care from their family physician, and over $80 \%$ of individuals with mental health problems received care solely within the primary care mental health system (CMHA, 2012). This clearly indicates that primary health care needs to be a key area of focus when looking to establish and build on our optimal mental health care strategies. This prescribed provision of mental health care within primary health care settings includes the identification and treatment of mental disorders, establishing prevention strategies, and ensuring that primary health care workers are equipped with adequate strategies and training to provide quality mental health care.

Some advantages to including mental health care within the primary health care model include: reduced stigma; improved access to care, including the ability to treat comorbidity and focus on detection, awareness, early intervention, or prevention of mental health problems; better treatment and follow-up including better physical and financial accessibility as well as acceptability; increased access to psychiatrists and other mental health professionals; improved social integration; and overall better health outcomes for individuals treated in primary health care (WHO, 2007; Clatney, MacDonald, \& Shah, 2012). Evidence has found that this improved access to services, and use of a stepped care model is particularly effective and beneficial for those with common mental disorders (NICE, 2011).

There are also larger scale financial benefits associated with the provision of better quality mental health care in primary care settings. Individuals experiencing mental health conditions represent the largest portion of individuals on disability support programs across Canada (CMHA, 2015). The treatment of depression and anxiety has been proven to be more effective through case management and use of a collaborative care team (Kendrick, 2013). Although there is an increased up front cost to more collaborative care models in primary health care settings, if effective treatment is provided, the result will ideally be fewer individuals on social supports, as well as less use of costly emergency health services.

Traditional treatment of common mental disorders has been heavily reliant on pharmaceuticals. There is growing evidence however in the benefits of expanding non-drug treatment options, based partly on self-help, and focusing on social support (Kendrick, 2013). Addressing positive mental health promotion concerns means aligning services with a holistic notion of health, and mental health. This is carried out largely by supporting individual resilience, establishing supportive environments, and acknowledging and focusing on the influence of the broader social determinants of health (CIHI, 2009). Primary health care is increasingly building its capacity to help respond to holistic mental health care needs with greater inclusion of various social determinants of health. For example, the increasing number of collaborative care teams comprised of diverse professional disciplines is allowing for a more in depth and holistic approach to healthcare and better acknowledging and addressing the social determinants. There is also an increase in health promotion strategies at the community and primary health care level that are addressing the social determinants as they relate not only to health but also mental health (CMHA, 2015). This shift in mindset and direction is also giving rise to a growing concern amongst primary care providers and users as to the gap between the current services provided and emerging evidence as to what works in mental health care. This has led to a current call to attention for increased support and resources in order to improve the quality of care provided (CMHA, 2012). Despite some of the innovations occurring in primary health care challenges persist and are making it problematic to implement services that respond to the scope of mental health needs.

\section{Challenges to Primary Mental Health Care}

Although primary health care is the ideal location for the provision of optimal mental health care, there are still numerous challenges and barriers to overcome. The health care system is such a large and multi-faceted organism, thus entailing many interlacing and intricate challenges, including contextual, systemic, organizational, and individual factors to contend with.

\section{Medical Focus}

As was previously discussed in the presentation of definitions of health, there are presently shortcomings in how we regard optimal health, and current definitions have lent to the medicalization of society (Godlee, 2011; Huber et al., 2011; Manwell et al., 2015). This identification of the limitations of the medical model represents another major barrier or challenge to overcome in order to provide optimal mental health care. The medical dominance of the mental health field has resulted in an increased focus on mental illness, particularly related to service, access, and stigma, rather than focusing on supporting the development of positive mental health (CIHI, 2009). It has also resulted in an increased level of pathologizing mental health concerns. Although we are beginning to see a shift toward a more holistic conception of health care, the traditional medical model of health does a poor job of recognizing and acting on the broader social determinants of health.

\section{Attitudes and Comfort Levels}

Treatment of these common mental disorders within the primary health care setting has proven to be significantly more time consuming relative to the treatment of other health concerns, adding to the reluctance or discomfort in providing care for mental health problems within the medical community.

Concerns regarding the attitudes and comfort levels of primary health care workers toward mental health is another significant barrier to overcome in providing optimal mental health care (WHO, 2007). There is a tendency among family physicians to understand depression as being reactive or internal, resulting in uncertainty as to treatment direction when derived from negative life events or difficulties (Kendrick, 2013). There are presently relatively low rates of detection and treatment for common mental disorders. Investigation into why there is often hesitation amongst family physicians in initiating treatment even once a common mental disorder has been detected has been identified as necessary (Craven \& Bland, 2013).

Ideologies and beliefs at a societal level also affect the delivery of mental health care. Many Canadians still show similarities with traditional American attribution of success or failure to individual qualities and initiative (Raphael, 2009). These beliefs can make it harder to relay the importance of government supports of the social determinants of health in order to provide better treatment for mental health concerns. Family physicians generally perceive this need to consider 
social factors as well, but find themselves often uncertain of how to do so in practice (Kendrick, 2013). Training can be geared toward addressing any reluctance of primary health care staff to working with people with mental disorders.

\section{Training and Resources}

There is also a need for increased and improved training for primary health care staff regarding mental disorder detection and treatment (WHO, 2007). Evidence suggests that a primary health care provider's lack of training in mental health, along with staffing models, access to mental health resources and specialists, and a patient's lack of insurance all contribute to patients with mental health problems not receiving adequate mental health care (Parliament of Canada, 2004; Brown, 2011).

This decreased public spending on health care not only affects individual health insurance, but also contributes to a general lack of resources and difficulty in securing stable funding for collaborative care, which presents another challenge to providing optimal mental health care in the primary care setting. A common response from family physicians regarding areas of improvement was the need to increase mental health resources, such as human resources, and mental health budgets (Clatney, MacDonald, \& Shah, 2008).

The traditional fee-for-service payment plans have also played a significant role in this barrier to the implementation of collaborative mental health care. We are beginning to see changes in the remuneration models towards roster based funding models, or blended capitation models (Kralj \& Kantarevic, 2012). However the physician centered payment models still pose challenges to the compensation of nonphysician providers and collaborative activities (Mulvale \& Bourgeault, 2007).

\section{An Overburdened System}

The commodity of time and the general overburdened nature of the health care system is another deterrent to providing more holistic and extensive collaborative care teams. One of the key components of the successful delivery of mental health care in the primary health care setting is the availability of mental health professionals to the primary care staff for consultation and supervision (WHO, 2007). Family physicians often report difficulties in accessing these mental health professionals and specialists, particularly psychiatrists, for such consultations or patient referrals (Clatney, MacDonald, \& Shah, 2008). This stifled access to mental health specialists is then compounded by the existing high demand for primary health care services, creating longer wait times for care and a decreased capacity for attentive, ongoing care.

The current remuneration models involving fee-forservice are also not conducive to dealing with mental health patients, who may require more time and attention. The quality of treatment is often deemed as inadequate regarding followup and monitoring (Craven \& Bland, 2013). Increased focus on collaborative care interventions and case management would likely address this.

At a macro level, the operation of the economic and political systems provides additional barriers to improving this system. Common with other liberal political economies, the government holds a more limited role in assuring citizen wellbeing, and is often reluctant to bring about legislation that will assure employment, housing, and income security
(Raphael, 2009). Not only does this cautious spending directly affect the ability of the healthcare system to create more collaborative care interventions and increased case management, but it also negatively affects patient populations in terms of the social determinants of health. This negative influence on the social determinants of health leads to further burden on the healthcare system with increased usage and more complex health concerns.

\section{Discussion}

There clearly exists an interest from all implicated parties to provide better mental health care within the primary health care setting. However, despite increased attention, training, and resources, there is still a general sense of inadequacy or uncertainty of competency within primary health care providers around treating common mental disorders and mental health problems.

Seemingly adjacent to this growing awareness and concern for mental health is the growing awareness of the importance of considering the social determinants of health. If there is a growing concern over the social determinants of health and acknowledging health as a holistic concept, then why aren't we concerting more resources toward allowing primary care providers to directly address these social determinants? Efforts are being made to come up with a more holistic conception of overall health. A transdomain model of health is being used to inform this more comprehensive definition incorporating the subcomponents of health which include physical, mental, and social health (Manwell et al, 2015). It would be immensely beneficial to blend these areas of focus more, and create a model of mental health care more concretely working with addressing the social determinants of health and mental health.

Ontario has been taking steps in the right direction in terms of providing funding for health promotion addressing the social determinants through the establishment of specific strategies such as the Poverty Reduction Strategy, the Healthy Eating and Active Living Strategy, the Eating at Home Strategy, among others (CMHA, 2012). We are even beginning to see more mental health promotion through strategies such as the Mental Health and Addictions Strategy (CMHA, 2015). Mental health promotion programs and policies represent a gainful step as they generally address the individual, their relation to their community, and the broader social environment they inhabit (CMHA, 2015).

This rounded consideration of the individual, community, and society is what the social determinants of health so nicely addresses. As was previously detailed, the determinants can affect health positively or negatively and include environmental, social, and biological factors that all contribute to an individual's wellbeing (Mikkonen \& Raphael, 2010). Government policies and programs primarily influence the majority of these factors. Some countries provide full coverage for all health care, including medications, dental care, and home care. However policies also largely affect other determinants such as poverty, housing, food, employment, and integration within society. Many developed nations go further than the health care coverage to also ensure these other determinants are being met. Unfortunately, Canada has one of the highest rates of family poverty amongst developed countries, and needs for access to food and housing are not being met for many (CPHA, 2015). This lack of good social 
determinants of health has been proven to result in adverse health outcomes (CPHA, 2015). It also places these individuals into positions of inequity, limited opportunity, unstable employment, and social isolation within society (Raphael, 2009). The negative ramifications can then lead to, or exacerbate existing, mental health problems.

The Canadian Mental Health Association (2015) provides us with some areas of focus for policy formation as well as health promotion addressing key social determinants that relate to mental health and the establishment of an environment for optimal mental health care. They include, the promotion of social inclusion, ensuring that all individuals live free from discrimination and violence, and that all individuals have access to economic resources. Of course these can be further disseminated to create more solid policy strategies, however what can be done to tackle this same aim at the primary care level? How can we encourage primary care providers to directly address the social determinants of health?

One such model that is coming into prominence in the United States that skirts this dependence on government policy is the Health Leads initiative. Stated in their vision is the aim for a healthcare system that acknowledges and answers all patients' basic resource needs as being a standard in the delivery of quality care (Health Leads, 2015). Essentially, they work alongside primary care providers to integrate patient resource screening and navigation into the care process. Health Leads trains university students, most commonly medical students, to volunteer as representatives in primary care offices to meet with patients and utilize a screening process largely informed by the social determinants of health in order to get a fuller picture of the patient and their environment. The program then enables physicians and primary care staff to provide more than just the typical pharmaceutical treatment, such as prescribing basic resources like food and heat, which the Health Leads representative then helps them to access.

An added benefit to the model used by Health Leads is that it familiarizes the student volunteers, and potential future doctors or health care workers, with the issues the social determinants of health acknowledge which will better equip them to serve their future patients and operate from a holistic perspective. The founder of Health Leads is a strong advocate for the creation of health systems that keep populations healthy rather than solely treating illness, and this adherence to social determinants of health absolutely plays into that. Although the focus of this program is on improving overall health, it no doubt is an extremely effective and viable solution to improving the overall treatment of mental health as well. The model can be expanded on within primary care teams to prescribe other needs such as housing, education, employment, and even social engagement. This social prescribing model is gaining popularity in the UK with programs such as exercise on prescriptions, prescriptions for learning, and arts on prescriptions taking off (Friedli et al, 2008). There is evidence suggesting this broader, more holistic framework depicted in social prescribing, emphasizing factors such as personal experiences, relationships, and social conditions, may be more compatible in tending to mental wellbeing and common mental disorders than a traditional medical model approach (Friedli et al., 2008; Rogers \& Pilgrim, 1997). This direct acknowledgement of the social determinants of health strengthens primary care's response to the biological, social, and psychological factors of health and mental health, thus improving treatment delivery for common mental disorders.

\section{Conclusion}

It is clear how pivotal of a role the social determinants of health play, as well as the importance of treating the biological, psychological, and social aspects of an individual in providing optimal mental health care. Primary health care has been identified as a major junction in the delivery of this quality mental health care. We need to continue to identify barriers to providing this high level of care within the primary health care system, as well as working toward new models of care delivery that better consider the effects of the various social determinants of health and mental health.

The solutions and initiatives discussed provide evidence of the effectiveness of these more holistically minded approaches to care. It is thus vital to continue the trend toward collaborative care models in primary health care, and push for the adoption of this type of collaboration at a larger service provision, and governmental policy level as well.

\section{References}

Bhugra, D., Till, A., \& Sartorius, N. (2013). What is mental health? International Journal of Social Psychiatry, 59(1), 3-4.

Braveman, P., Egerter, S., \& Mockenhaupt, R. (2011). Broadening the focus: the need to address the social determinants of health. American Journal of Preventative Medicine 40(1), S4-S18.

Brown, R. (2011). A Primary Care Provider's Description of Mental Health Services. (Doctoral dissertation, University of Phoenix).

Canadian Institute for Health Information. (2006). PanCanadian primary health care indicators. Report 1, Volume 1. Ottawa: CIHI.

Canadian Institute for Health Information. (2009). Improving the Health of Canadians: Exploring Positive Mental Health. Ottawa: CIHI.

Canadian Mental Health Association. (2012). Improving Primary Care Mental Health Services. British Columbia Division: CMHA. Retrieved from https://www.cmha.bc.ca/get-informed/publicissues/primarycare

Canadian Mental Health Association. (2015). Mental Health: Your Mental Health. CMHA. Retrieved from https://www.cmha.ca/mental-health/your-mental-health/

Canadian Mental Health Association. (2015). Services and Support: Income. Ontario: CMHA. Retrieved from https://ontario.cmha.ca/mental-health/services-andsupport/income/

Canadian Public Health Association (n.d.). What are the Social Determinants of Health? Retrieved from http://www.cpha.ca/en/programs/socialdeterminants/frontlinehealth/sdh.aspx

Centre for Addiction and Mental Health (CAMH), Centre for Health Promotion, Canadian Mental Health Association (CMHA) Ontario, Health Nexus, \& Ontario Public Health Association (OPHA). (2008). Mental Health Promotion in Ontario: A Call to Action. November 2008. Clatney, L., MacDonald, H., \& Shah, S. (2008). Mental health 
care in the primary care setting: Family physicians' perspectives. Canadian Family Physician, 54(6), 884889.

Craven, M., \& Bland, R. (2013). Depression in primary care: Current and future challenges. Canadian Journal of Psychiatry, 58(8), 442-448.

Culpepper, L., Judd, C., Moller, M., Nemeroff, C., Rapaport, M., \& Ciraulo, D. (2006). Clinicians on the front line: Active management of depression and anxiety in primary care. Journal of the American Academy of Physician Assistants, Suppl, 4-21.

Friedli, L., Jackson, C., Abernethy, H., \& Stansfield, J. (2008). Social prescribing for mental health - a guide to commissioning and delivery. Lancashire: Care Services Improvement Partnership North West.

Godlee, F. (2011). What is health? BMJ, 343:d4817 Government of Canada. (2006). The Human Face of Mental Health and Mental Illness in Canada. Ottawa: Minister of Public Works and Government Services Canada.

Health Council of Canada. (2005). Primary health care. A background paper to accompany health care renewal in Canada: Accelerating change. Toronto: Health Council of Canada.

Health Education Authority. (1997). Mental health promotion: A quality framework. London: HEA.

Health Leads. (2015). Solutions for Healthcare Providers. Retrieved from https://healthleadsusa.org

Huber, M., Knottnerus, J. A., Green, L., van der Horst, H., Jadad, A. R., Kromhout, D., Leonard, B., Lorig, K., Loureiro, M. I., van der Meer, J. W. M., Schnabel, P., Smith, R., van Weel, C., \& Smid, H. (2011). How should we define health? BMJ, 343:d4163.

Kates, N., Mazowita, G., Lemire, F., Jayabarathan, A., Bland, R., Selby, P., Isomura, T., Craven, M., Gervais, M., \& Audet, D. (2011). The evolution of collaborative mental health care in Canada: a shared vision for the future. Canadian Journal of Psychiatry, 56(5), Insert 1-10.

Kendrick, T. (2013). Depression in primary care: what more do we need to know? Canadian Journal of Psychiatry, 58(8), 439-441.

Kralj, B., \& Kantarevic, J. (2012). Primary care model enrolment and hospital length of stay in Ontario: patient rostering associated with reduced length of stay, significant health system savings. Ontario Medical Review, September 2012, 16-19.

Manwell, L., Barbic, S., Roberts, K., Durisko, Z., Lee, C., Ware, E., \& McKenzie, K. (2015). What is mental health? Evidence towards a new definition from a mixed methods multidisciplinary international survey. $B M J$ Open 2015; 5:e007079. doi: 10.1136/bmjopen-2014007079

Marmot, M., Allen, J., Bell, R., Bloomer, E., \& Goldblatt, P. (2012). WHO European review of social determinants of health and the health divide. Lancet, 380(9846), 15-21.

Maslow, A. (1968). Towards a psychology of being. New
York: Van Nostrand. Mikkonen, J., \& Raphael, D. (2010). Social Determinants of Health: The Canadian Facts. Toronto, Canada: York University School of Health Policy and Management.

Mitchell, P., Wynia, M., Golden, R., McNellis, B., Okun, S., Webb, E., Rohrbach, V., \& Von Kohorn, I. (2012). Core Principles \& Values of Effective Team-Based Health Care. Discussion Paper, Institute of Medicine, Washington, DC. Retrieved from https://nationalahec.org/pdfs/VSRT-Team-Based-CarePrinciples-Values.pdf

Mulvale, G., \& Bourgeault, I. (2007). Finding the right mix: How do contextual factors affect collaborative mental health care in Ontario? Canadian Pubic Policy, 33(1), 49-64.

National Institute for Health and Care Excellence. (2011). Common Mental Health Disorders. UK: NICE. Retrieved from https://www.nice.org.uk/guidance/cg123

Parliament of Canada (2004). Interim report on the mental health, mental illness and addiction: Issues and options for Canada (Report No. 3). Ottawa, Canada: Standing Senate Committee on Social Affairs, Science and Technology.

Primary Care Companion. (2008). Treating depression and anxiety in primary care. Journal of Clinical Psychiatry, 10(2), 145-152.

Raphael, D. (2009). Restructuring society in the service of mental health promotion: are we willing to address the social determinants of mental health? International Journal of Mental Health Promotion, 11(3), 18-31.

Rogers, A. \& Pilgrim, D. (1997). The contribution of lay knowledge to the understanding and promotion of mental health. Journal of Mental Health, 6(1), 23-35.

Sartorius, N. (2002). Fighting for mental health. Cambridge: Cambridge University Press.

Society for Health Education and Promotion Specialists. (1997). Ten elements of mental health, its promotion and demotion: Implications for practice. London: SHEPS.

Whitebird, R., Solberg, L, Margolis, K., Asche, S., Trangle, M., \& Wineman, A. (2013). Barrier to improving primary care of depression: perspectives of medical group leaders. Qualitative Health Research, 23(6), 805814.

Whiteford, H., Degenhardt, L., Rehm, J., Baxter, A., Ferrari, A., Erskine, H., Charlson, F., Norman, R., Flaxman, A., Johns, N., Burstein, R., Murray, C., \& Vos, T. (2013). Global burden of disease attributable to mental and substance use disorders: findings from the Global Burden of Disease Study 2010. Lancet, 382(9904), 15401542.

World Health Organization. (2006). Constitution of the World Health Organization. Retrieved from www.who.int/governance/eb/who_constitution_en.pdf

World Health Organization. (2014). Mental health: strengthening our response, Fact sheet No. 220. Geneva: WHO. Retrieved from http://www.who.int/mediacentre/factsheets/fs220/en/ 